\title{
molecules
}

ISSN 1420-3049

http://www.mdpi.org

Editorial

\section{Non-Open Access and Its Adverse Impact on Molecules}

\section{Shu-Kun Lin}

Molecular Diversity Preservation International (MDPI), Matthaeusstrasse 11, Basel CH-4057, Switzerland; Tel. ++41 79322 3379, Fax: ++41 61302 8918, E-mail: lin@mdpi.org, http://www.mdpi.org/lin

Received: 10 July 2007 / Published: 16 July 2007

In 2005 and 2006 papers were published in MDPI journals in both non-Open Access (non-OA) and Open Access (OA) form [1,2]. In 2005, 70 papers (just under $50 \%$ of the total) were published in the password protected (non-Open Access) area of Molecules. Not undexpectedly, this reduced number of Open Access papers had a significant influence on the impact factor of Molecules, which was reduced from 1.113 in 2005 to 0.841 in 2006 . According to the methods used in calculating impact factors, the number of papers published in 2005 and the number of citations were used in the calculation of the 2006 impact factor. The statistical data for non-OA and OA are presented in Table 1.

Table 1. Open Access (OA) and non-Open Access (non-OA) papers published in Molecules in 2005 [3].

\begin{tabular}{|l|c|c|c|}
\hline & Number of papers $^{1}$ & Number of citations $^{2}$ & Average citation $^{-1}$ \\
\hline Non-OA $^{3}$ & 70 & 43 & 0.614 \\
\hline OA & 74 & 90 & 1.216 \\
\hline
\end{tabular}

${ }^{1}$ Only research papers and review papers are counted. Other materials such as editorials, are not included. ${ }^{2}$ Up to 13 July 2007, according to Web of Sciences. ${ }^{3}$ By April 2007, all the non-OA papers were changed to Open Access.

Like Molecules, in 2005 another MDPI journal, International Journal of Molecular Sciences, also published a significant percentage of papers in its password protected (non-Open Access) area. The impact factor of International Journal of Molecular Sciences was also reduced from 1.467 in 2005 to 0.679 in 2006. The statistical data for non-OA and OA are presented in Table 2.

As shown in Tables 1 and 2, the non-Open Access papers and their obviously lower citation numbers appear to be the main reason behind the reduced impact factors of these two journals, where almost half of the papers published were in non-OA form. 
Table 2. Open Access (OA) and non-Open Access (non-OA) papers published in International Journal of Molecular Sciences in 2005 [3].

\begin{tabular}{|l|c|c|c|}
\hline & Number of papers $^{1}$ & Number of citations $^{2}$ & Average citation \\
\hline Non-OA $^{3}$ & 11 & 5 & 0.455 \\
\hline OA & 13 & 19 & 1.462 \\
\hline
\end{tabular}

${ }^{1}$ Only research papers and review papers are counted. Other materials such as editorials, are not included. ${ }^{2}$ Up to 13 July 2007, according to Web of Sciences. ${ }^{3}$ By April 2007, all the non-OA papers were changed to Open Access.

As a contrast, the MDPI journal Sensors (http://www.mdpi.org/sensors/) published only a very small number of non-Open Access papers in 2005, and its impact factor increased slightly from 1.208 in 2005 to 1.373 in 2006.

Since April 2007, we have granted full Open Access to all the papers published in all MDPI journals in 2005 and 2006, and in 2007, we have published papers exclusively in Open Access form. We expect this will be reflected in an increase in impact factors come the next evaluation.

As always, we welcome our reader's opinions on these matters [4]. Messages with a suitable Subject header should be sent to lin@mdpi.org.

\section{Acknowledgements}

I am grateful for comments and corrections made by Dr. Ricardo Aparicio and Dr. Derek McPhee.

\section{References and Notes}

1. Lin, S.-K. Open Access and Author's Open Choice. Molecules 2005, 10, 583-584 (PDF format 16 $\mathrm{K}$ at http://www.mdpi.org/molecules/papers/10060583.pdf; HTML format at http://www.mdpi.org/molecules/html/10060583.htm).

2. Lin, S.-K. Delayed Open Access or Permanent Non-Open Access. Molecules 2006, 11, 496-497 (PDF format $15 \mathrm{~K}$ at http://www.mdpi.org/molecules/papers/11070496.pdf; HTML format at http://www.mdpi.org/molecules/html/11070496.htm).

3. The raw data in Excel format is available upon request.

4. For more information, visit the www.mdpi.org/oaj-supports.htm website.

(C) 2007 by Molecular Diversity Preservation International (MDPI). 